\title{
Utilização de métodos contraceptivos entre as usuárias da rede pública de saúde do municipio de Maringá-PR
}

\author{
Contraceptive methods in the public health service (“Sistema Único de Saúde”) in Maringá-PR
}

Jovita Maria Matarezi de Souza ${ }^{1}$, Sandra Marisa Pelloso ${ }^{2}$, Nelson Shozo Uchimura ${ }^{3}$, Fernando de Souza ${ }^{4}$

\section{RESUMO}

Objetivo: avaliar os métodos contraceptivos adotados e o perfil das usuárias da rede pública de saúde do município de Maringá-PR, em relação às orientações para o seu uso, indicações, contra-indicações e razões para interrupção dos métodos. Métodos: estudo transversal, descritivo, obtido por 284 entrevistas domiciliares de mulheres selecionadas das 62 equipes do Programa de Saúde da Família, mediante consentimento livre e esclarecido. O questionário foi aprovado pelo Comitê de Ética em Pesquisa envolvendo seres humanos da Universidade Estadual de Maringá. Os questionários utilizados foram prétestados e então aplicados, interessando para este estudo as seguintes seções: caracterização da entrevistada; indicadores socioeconômicos; métodos contraceptivos. Análise estatística das distribuições de freqüências, pelo Statistical Package for the Social Sciences, versão 12.0. Resultados: a maioria das mulheres eram de etnia branca, casadas, tinham idade entre 35 e 49 anos, escolaridade em nível médio, exerciam trabalho não remunerado e foram classificadas na classe econômica D e E. Quanto aos hábitos, 22,5\% eram fumantes e 4,9\% usuárias de bebida alcoólica. A pílula foi adotada em 50,3\%, preservativo em $28,1 \%$ e laqueadura em $32 \%$. A orientação quanto ao uso de métodos anticoncepcionais geralmente foi dos profissionais da área de saúde. Os motivos de interrupção dos métodos foram: desejo de engravidar, preferência por método definitivo e efeitos colaterais da pílula. O fator de risco mais prevalente para uso de pílula foi o tabagismo. Apenas 35,9\% das entrevistadas iniciaram o uso da pílula após consulta prévia e quase a mesma proporção, 33,6\%, não a fizeram antes do início de seu uso. Conclusões: observou-se que as indicações do uso do método contraceptivo e as orientações realizadas por profissionais da área de saúde foram satisfatórias, apesar das elevadas taxas de laqueadura e de detecção de usuárias de pílulas com contra-indicações relativas, com mais de cinco anos de uso.

PALAVRAS-CHAVE: Anticoncepção; Saúde da mulher; Medicina reprodutiva; Serviços de planejamento familiar

\section{ABSTRACT}

Purpose: to evaluate the contraceptive methods adopted by the public health system of Maringá County, Paraná, regarding the orientations for using them, indications, contraindications and reasons for interrupting these methods, as well as the profile of the female users. Methods: transversal descriptive study, performed through 284 home interviews with women selected from the 62 groups of the Family Health Program, after their free and informed consent, and after the questionnaire had been approved by the Ethics in Research Committee involving human beings of the State University of Maringá (Universidade Estadual de Maringá - UEM). Before applying the questionnaires, they were pretested, focusing on the following sections: characterization of the interviewee, socioeconomical factors and contraceptive methods. Results were analyzed using the Statistical Package for the Social Sciences software 12.0 version. Results: most women were white, married, between 35 and 49 years old, with high school education, working without salary, and from D and E economical classes. Of them, $22.5 \%$ were smokers and $4.9 \%$ alcohol users. Contraceptive pills were adopted by $50.3 \%$ of the women; condom by $28.1 \%$ and tubal ligature by $32 \%$, following, in general, the health professional orientation. Reasons for interrupting the contraceptive methods were the wish to get pregnant, preference for a permanent method, and also because of the side effects of the pill. Smoking was the most prevalent risk factor for pill use. Only $35.9 \%$ of the interviewed women started using

1 Professora Assistente da Disciplina de Ginecologia e Obstetrícia do Departamento de Medicina da - Universidade Estadual de Maringá - UEM - Maringá (PR), Brasil.

2 Professora Associada do Departamento de Enfermagem - UEM, Professora do Programa de Pós-graduação em Ciências da Saúde - Universidade Estadual de Maringá - UEM - Maringá (PR), Brasil.

3 Professor Adjunto da Disciplina de Ginecologia e Obstetrícia do Departamento de Medicina da UEM e Chefe da Divisão da Área de Ginecologia e Obstetrícia do Hospital Universitário de Maringá (PR), Brasil.

4 Professor Adjunto da Disciplina de Cirurgia do Aparelho Digestivo do Departamento de Medicina da Universidade Estadual de Maringá - Universidade Estadual de Maringá - UEM - Maringá (PR), Brasil.

Correspondência: Jovita Maria Matarezi de Souza

Av. Dr. Luiz Teixeira Mendes, 2136 - 87015-000 - Maringá - PR - Telefone: (44) 3224-3949 - e-mail: jovitamatarezi@uol.com.br 
the pill after a previous medical visit, and almost in the same proportion, 33.6\%, without visit before starting to use it. Conclusions: it was observed that the indications of the contraceptive method to be used, and the orientations accomplished by the health professionals were satisfactory, despite the high levels of tubal ligature and the detection of relative contraindications for pill users with more than 5 years of use.

KEYWORDS: Contraception; Women's health; Reproductive medicine; Family planning services

\section{Introdução}

Dentre os vários aspectos da saúde reprodutiva, a anticoncepção adquire papel inigualável e seu uso de forma inadequada implica vários agravos à saúde da mulher, como gravidez indesejada, gravidez na adolescência, abortamentos ilegais e até mesmo aumento na mortalidade materna. Também não se pode desprezar as conseqüências geradas por uma cesariana indicada às vezes exclusivamente para se realizar uma laqueadura, o que implica potenciais riscos de morbidade (física e psicológica) e até de mortalidade destas intervenções sobre o processo de nascimento ${ }^{1}$.

Realizar então o planejamento familiar é uma das prerrogativas da Medicina Preventiva, tendo como objetivos reduzir a morbidade e a mortalidade materno-infantil resultantes, principalmente, de algum abortamento provocado ou de uma gestação de alto risco. Números apresentados no relatório de 1997 do Fundo Populacional das Nações Unidas, demonstram a incontestável necessidade de sua implantação: 350 milhões de mulheres no mundo não têm acesso a métodos contraceptivos modernos e, por ano, 600 mil morrem de causas relacionadas a gestações, das quais 75 mil são causadas por complicações de abortamentos provocados ${ }^{2}$.

Em relação à assistência em anticoncepção no Brasil, na Pesquisa Nacional de Demografia e Saúde (PNDS) de 1996 o método contraceptivo por laqueadura tubária representou $40 \%$ dos casos e a pílula anticoncepcional, $21 \%$. A maioria das mulheres submetidas à laqueadura se encontravam dentro das classes de menores índices de escolaridade e nível econômico ${ }^{3}$. No estado de São Paulo, em 1988, observou-se que mais da metade das mulheres referiram ter sido submetidas à laqueadura antes dos 30 anos $^{4}$. Também é significante e já discutido por alguns autores que a laqueadura não é isenta de conseqüências hormonais ${ }^{5}$, e a alta prevalência de laqueadura tubária contribui para aumentar o número de cesáreas desnecessárias $^{4,6}$, e estas por sua vez contribuem para aumen- tar os índices de mortalidade materna ${ }^{1,7-9}$. Outro aspecto muito relevante neste contexto é que, em 1990, observou-se que metade dos nascimentos vieram de gestações não planejadas. Além disto, segundo o Instituto Brasileiro de Geografia e Estatística (IBGE), no censo do ano 2000, vem ocorrendo aumento de gestações na faixa etária entre 15 e 19 anos, principalmente nas regiões mais pobres do país e entre as mulheres com menor nível de escolaridade, explicado pelo início precoce da puberdade e atividade sexual ${ }^{10,11}$. A desinformação da população associada à dificuldade de acesso aos serviços de saúde de qualidade e limitações dos próprios métodos contraceptivos configuram-se como os fatores preponderantes a caracterizar a discutivel qualidade da assistência em planejamento familiar no Brasil. Segundo a Norma Operacional de Assistência à Saúde (NOAS-2001), as ações do pla-nejamento familiar fazem parte da atenção básica e são responsabilidade da gestão municipal da saúde da mulher ${ }^{12}$, mas, infelizmente, muitos municípios não implantam estratégias adequadas para fornecimento de anticoncepcionais para a população, programa educativo e de aconselhamento para livre escolha do método e, assim, garantir o acompanhamento das usuárias ${ }^{10,12}$.

Também é importante acrescentar que as ações em anticoncepção são atreladas à prevenção de DST, dentre as quais a síndrome da imunodeficiência adquirida, agravos estes que vêm apresentando tendência de crescimento entre as mulheres e jovens ${ }^{11,13}$. O uso de métodos adequados e eficazes associados à prevenção de DST esbarram em dificuldades de aceitabilidade, taxa de descontinuação e o próprio preconceito que o tema DST propicia ${ }^{14,15}$. Todas as mulheres, solteiras, casadas, com ou sem filhos, grávidas ou não, deveriam ter acesso aos cuidados de saúde dirigidos à prevenção, detecção precoce, tratamento e reabilitação dos principais agravos à saúde a que estão expostas, dentre estes, as informações e orientações em contracepção ${ }^{16}$.

Em torno destas considerações, o presente trabalho tem como objetivo avaliar a utilização de métodos contraceptivos das mulheres usuárias dos serviços de saúde da rede pública do municí- 
pio de Maringá-PR, sob o ponto de vista da usuária, através de inquérito populacional.

\section{Métodos}

Estudo descritivo, de corte transversal, aplicado por meio de inquérito populacional aplicado no município de Maringá-PR, a mulheres cadastradas no Programa de Saúde da Família (PSF), no ano de 2004.

A população estimada para Maringá, pelo censo de 2000 do IBGE, foi de 288.465 habitantes, e a projeção para 2003, de 303.243 habitantes. A estimativa de mulheres em idade reprodutiva, 10-49 anos segundo a Organização Mundial da Saúde, residentes na cidade de Maringá-PR, pela projeção do último censo para o ano de 2003, foi de 99.783 mulheres ${ }^{17}$. A rede pública de saúde no município de Maringá faz parte da $15^{\mathrm{a}} \mathrm{Re}-$ gional de Saúde e compõe-se de 23 Unidades Básicas de Saúde (UBS). A população usuária das UBS e as equipes de PSF estão distribuídas em cinco regionais, que são de Pinheiros, Quebec, Iguaçu, Tuiuti e Zona Sul, que correspondem à área geográfica respectiva. No período do estudo o município estava cadastrado junto ao Ministério da Saúde com 62 equipes de PSF. A seleção dos endereços nos bairros, para as entrevistas, foi realizada em função do número total das 62 equipes de PSF distribuídas em 5 regionais, englobando as diferentes regiões da cidade de forma aleatória e estratificada, obtendo-se 284 entrevistas. Foi redigido um texto em forma de consentimento livre e esclarecido. A proposta do projeto foi registrada no Comitê Permanente de Ética em Pesquisa envolvendo Seres Humanos da Universidade Estadual de Maringá, sendo submetida à apreciação e obtendo parecer favorável, sob o n⿳⺈ $201 / 2004$.

Os questionários utilizados foram pré-testados e então aplicados, interessando para este estudo as seguintes seções: caracterização da entrevistada, indicadores socioeconômicos e métodos contraceptivos.

Em relação ao tamanho da amostra, utilizou-se o modelo: $\mathrm{N}=(\mathrm{Z} / \mathrm{d})^{2} \cdot \mathrm{p}(1-\mathrm{p})$, onde $\mathrm{N}$ é o tamanho da amostra, $Z$ é a variável normal reduzida, p(1-p) é o desvio padrão amostral e d é o erro padrão ${ }^{18}$. Neste caso, o tamanho da amostra foi de 284 para um intervalo de confiança com 95\% e um erro padrão de $5,82 \%$. Os dados foram processados e submetidos a estudo estatístico utilizando-se o software SPSS (Statistical Package for the Social Sciences) versão 12.0. Inicialmente foi construída uma base de dados a partir dos dados coletados pelas entrevistadoras. Como primeiro passo efetuou-se o cálculo do coeficiente alfa de Cronbach e das correlações de Spearman e Kendall, a fim de garantir a consistência interna e sua precisão. O valor obtido do coeficiente alfa nas variáveis escalonadas foi 0,82 . A partir dos resultados observou-se a fidedignidade do instrumento, o que veio garantir a consistência e precisão de sua medida. Os resultados estão apresentados por meio de tabelas de distribuição de freqüências.

A variável classe econômica foi baseada no Critério de Classificação Econômica Brasil (CCEB) da Associação Brasileira de Empresas de Pesquisa $(\mathrm{ABEP}, 2004)^{19}$, composta por sete classes A1, A2, B1, B2, C, D e E e baseada na posse de bens de consumo. As classes A1, A2, B1 e B2 foram agrupadas às classes $\mathrm{B}$ e a classe $\mathrm{E}$ foi agrupada à classe $\mathrm{D}$, pois menos de $5 \%$ da amostra se enquadravam nessas categorias.

Os motivos pesquisados para a interrupção dos métodos contraceptivos foram baseados nas cinco categorias de Curtis e Hammerslough, que são: falha no uso, desejo de ficar grávida, efeito colateral, razões relacionadas ao uso (companheiro não gosta, contra-indicação, acesso/disponibilidade, queria método mais eficaz, inconveniente de usar/não gostou, custo e fatalismo) e outras ${ }^{20}$. No presente estudo, estas categorias foram agregadas e adaptadas para: 1- desejo de engravidar, 2preferência por método definitivo, 3- falha no uso, 4- efeito colateral, 5- dificuldades de acesso/custo e 6- outras.

Estes dados fazem parte de pesquisa mais ampla de caracterização da qualidade de assistência à saúde reprodutiva das mulheres usuárias dos serviços de saúde na rede pública do município.

\section{Resultados}

Foram analisados os resultados obtidos em 284 questionários das entrevistas realizadas. Observou-se $80,3 \%$ das mulheres do estudo eram de etnia branca, $66,5 \%$ casadas e $46,8 \%$ se encontravam na faixa etária entre 35 e 49 anos de idade. Quanto à escolaridade, $87 \%$ haviam chegado ao nível médio de ensino; 54,2\% exerciam atividade de trabalho não remunerada e 55,3\% eram pertencentes às classes econômicas D e E. Quanto aos hábitos, $22,5 \%$ eram fumantes e $4,9 \%$ faziam uso de bebida alcoólica (Tabela 1). 
Tabela 1 - Distribuição do número (n) e do percentual (\%) das entrevistadas segundo variáveis sócio-culturais-demográficas.

\begin{tabular}{|c|c|c|}
\hline Atributo & $\mathrm{n}$ & $\%$ \\
\hline \multicolumn{3}{|l|}{ Etnia } \\
\hline Branca & 228 & 80 , \\
\hline Não branca & 56 & 19, \\
\hline \multicolumn{3}{|l|}{ Estado civil } \\
\hline Casada & 189 & 66 \\
\hline Solteira & 77 & 27 , \\
\hline Separada & 12 & 4 \\
\hline Viúva & 6 & 2 \\
\hline \multicolumn{3}{|l|}{ Faixa etária } \\
\hline 10 a 14 anos & 4 & 1 \\
\hline 15 a 24 anos & 76 & \\
\hline 25 a 34 anos & 71 & \\
\hline 35 a 49 anos & 133 & 46 \\
\hline \multicolumn{3}{|l|}{ Escolaridade } \\
\hline $1^{\circ}$ grau incompleto & 22 & 7 \\
\hline $2^{\circ}$ grau completo & 247 & 87 , \\
\hline Superior completo & 15 & 5 \\
\hline \multicolumn{3}{|l|}{ Trabalho que executa } \\
\hline Não remunerado & 154 & \\
\hline Remunerado & 130 & \\
\hline \multicolumn{3}{|l|}{ Classe econômica* } \\
\hline$A e B$ & 21 & 7 \\
\hline C & 106 & \\
\hline De E & 157 & \\
\hline \multicolumn{3}{|l|}{ Tabagismo } \\
\hline Não fumantes & 220 & 77 \\
\hline Fumantes & 64 & 22 \\
\hline \multicolumn{3}{|l|}{ Etilismo } \\
\hline Não usuárias de bebidas alcoólicas & 170 & 59 \\
\hline Etilistas sociais & 100 & \\
\hline Usuárias de bebidas alcoólicas & 14 & \\
\hline
\end{tabular}

*Variável baseada no Critério Brasil de Classificação Econômica da Associação Brasileira de Estatística e Pesquisa (ABEP, 2004) ${ }^{19}$.

Constatou-se que dos métodos atualmente em uso, os classificados como pílula foram relacionados em 50,3\%, o condom em $28,1 \%$ e a laqueadura em $32 \%$. Os demais tipos, como contraceptivo injetável, preservativo feminino, espumas e geléias, aparecem em $1,7 \%$ das vezes e nenhuma mulher entrevistada usava diafragma, implantes ou endoceptivo (Tabela 2). Em relação aos métodos anticoncepcionais, as entrevistadas, para utilização do método natural, receberam orientações dos profissionais de saúde em $42,3 \%$ das vezes. Para utilização do condom, receberam, na mesma proporção, informações de parentes ou amigos e profissionais da área de saúde em $31,2 \%$ das vezes e, quanto ao uso de pílula, em $74 \%$ das vezes a orientação recebida foi através de profissionais da área de saúde (Tabela 3).

Tabela 2 - Distribuição do número (n) e do percentual (\%) das entrevistadas segundo o método contraceptivo em uso.

\begin{tabular}{lrr}
\hline Contraceptivos & $\mathbf{n}$ & $\%$ \\
\hline Cirúrgicos & 91 & 32,0 \\
$\quad$ Laqueadura & 6 & 2,1 \\
Vasectomia & 52 & 18,3 \\
Naturais* & 80 & 28,1 \\
Condom & 143 & 50,3 \\
Pílula & 6 & 2,1 \\
Mecânico - DIU & 0 & 0 \\
Diafragma & 0 & 0 \\
Norplant & 0 & 0 \\
Endoceptivo & 5 & 1,7 \\
Outros $^{* *}$ & &
\end{tabular}

${ }^{*}$ Métodos naturais - tabelinha, coito interrompido.

**Outros incluem: preservativo feminino, contraceptivo injetável, geléias e espermaticidas. A soma total excede $n=284$, por haver superposição (uso associado de dois ou mais métodos).

Tabela 3 - Distribuição do número (n) e do percentual (\%) dos métodos contraceptivos (natural, condom, pílula) segundo as categorias de quem recebeu orientação sobre como utilizar o método.

\begin{tabular}{lrrrrrr}
\hline & \multicolumn{7}{c}{ Método } \\
& Natural & \multicolumn{2}{c}{ Pílula } & \multicolumn{2}{c}{ Condom } \\
De quem recebeu orientação & \multicolumn{1}{c}{$\mathbf{n}$} & $\%$ & $\mathbf{n}$ & $\%$ & $\mathbf{n}$ & $\%$ \\
\hline Parentes e amigos & 8 & 15,4 & 25 & 31,2 & 15 & 6,7 \\
Professores, escola & 9 & 17,3 & 12 & 15,0 & 14 & 6,3 \\
Profissional de saúde* & 22 & 42,3 & 25 & 31,2 & 165 & 74,0 \\
Farmácia, revistas, folhetos & 5 & 9,6 & 7 & 8,8 & 23 & 10,3 \\
Ninguém & 8 & 15,4 & 11 & 13,8 & 6 & 2,7 \\
Total & 52 & 100 & 80 & 100 & 223 & 100 \\
\hline
\end{tabular}

*Médicos, enfermeiros.

Obs.: considerou-se o uso nos últimos 5 anos.

O motivo da interrupção dos métodos contraceptivos foram pesquisados, obtendo-se que, naquelas mulheres que se utilizavam métodos naturais, o desejo de engravidar foi o mais freqüente, em 31\%. Entre aquelas mulheres que se utilizavam do condom, a razão para interrupção, na maior parte das vezes, em $24,6 \%$, foi por ter sido submetida a método cirúrgico definitivo. No grupo de usuárias de pílula, em $23,8 \%$ a presença de efeitos colaterais foi a razão apontada para a interrupção do método (Tabela 4). 
Tabela 4 - Distribuição do número (n) e do percentual (\%) de métodos contraceptivos (natural, condom, pílula) de acordo com a razão dada para sua interrupção.

\begin{tabular}{|c|c|c|c|c|c|c|}
\hline \multirow[b]{3}{*}{ Razões para interrupção } & \multicolumn{6}{|c|}{ Método } \\
\hline & \multicolumn{2}{|c|}{ Natural } & \multicolumn{2}{|c|}{ Condom } & \multicolumn{2}{|c|}{ Pílula } \\
\hline & $\mathbf{n}$ & $\%$ & $\mathrm{n}$ & $\%$ & $\mathrm{n}$ & $\%$ \\
\hline Desejo de engravidar & 13 & 31,0 & 14 & 21,5 & 18 & 12,6 \\
\hline Preferência por método definitivo & 12 & 28,6 & 16 & 24,6 & 24 & 16,8 \\
\hline Falha no uso & 6 & 14,3 & 12 & 18,5 & 21 & 14,7 \\
\hline Efeito colateral & 0 & 0 & 9 & 13,9 & 34 & 23,8 \\
\hline Dificuldades de acesso, custo & 0 & 0 & 10 & 15,4 & 25 & 17,5 \\
\hline Outras (não lembra, não sabe) & 11 & 26,2 & 4 & 6,1 & 21 & 14,7 \\
\hline Total & 42 & 100 & 65 & 100 & 143 & 100 \\
\hline
\end{tabular}

A soma dos 3 métodos (natural, condom, pílula) apresentados e considerados para interrupção foi 250 (método natural $n=42$, condom $n=65$, pílula $n=143$ ).

Em se tratando de contra-indicações ou fatores de risco para o uso de pílula anticoncepcional, observou-se que 49,3\% das mulheres entrevistadas não apresentaram nenhum fator de risco, mas por outro lado, muitas delas apresentavam mais de um fator de risco, sendo o mais freqüente o tabagismo, constatado em $23,3 \%$. Em relação ao tempo de uso de pílula, observou-se que $29,6 \%$ vêm utilizando-se da pílula por mais de cinco anos e igual proporção das mulheres entrevistadas a utilizaram por até um ano. Apenas 35,9\% das entrevistadas iniciaram o uso da pílula após consulta prévia e quase a mesma proporção, 33,6\%, não a fizeram antes do início de seu uso (Tabela 5).

Tabela 5 - Distribuição do número (n) e do percentual (\%) de mulheres usuárias de pílula segundo contra-indicações, tempo de uso e consulta prévia.

\begin{tabular}{lrc}
\hline Contraceptivo oral - pílula & $\mathbf{n}$ & $\%$ \\
\hline Contra-indicações & 110 & 49,3 \\
Sem fatores de risco & 15 & 6,7 \\
Pressão Alta & 3 & 1,3 \\
Diabetes & 3 & 1,3 \\
Convulsões & 22 & 9,9 \\
Cefaléia forte, constante & 18 & 8,1 \\
Varizes (com dor, quente e inchaço) & 52 & 23,3 \\
Tabagismo & 0 & 0 \\
Doença cardíaca & 0 & 0 \\
Hepatite & 223 & 100 \\
Total & & \\
Tempo de uso & 66 & 29,6 \\
Até 1 ano & 39 & 17,5 \\
+ de 1 até 3 anos & 52 & 23,3 \\
+ de 3 até 5 anos & 66 & 29,6 \\
+ de 5 anos & 223 & 100 \\
Total & & \\
Consultou para iniciar a pílula & 80 & 35,9 \\
Sim & 75 & 33,6 \\
Não & 68 & 30,5 \\
Não se lembra, não soube informar & 223 & 100 \\
Total & & \\
\hline
\end{tabular}

\section{Discussão}

Os resultados deste estudo e os dados da literatura mostram a necessidade de se evoluir no aspecto de saúde integral da mulher, incluindo e respeitando os múltiplos aspectos da anticoncepção em saúde reprodutiva. Em relação ao planejamento familiar, observa-se elevado uso dos métodos anticoncepcionais. A qualidade dessa prática, entretanto, é discutivel ao menos em relação aos principais métodos analisados e mais utilizados pelas mulheres, a pílula anticoncepcional, laqueadura tubária e o condom. Neste estudo, constatou-se elevada prevalência de uso de pílula anticoncepcional, relatado por $50,3 \%$ das entrevistadas, sendo submetidas à laqueadura $32 \%$ e em uso de condom $28,1 \%$.

A laqueadura tubária, método de esterilização cirúrgica feminina, foi o método definitivo mais utilizado e o terceiro método entre os demais, sendo que este predomínio confirma a tendência nacional ${ }^{3-5,21}$. Por ter caráter definitivo, a laqueadura deveria ser método de exceção e não o mais prevalente. Alguns aspectos negativos, como alta prevalência e dificuldades em critérios de indicação, tornam-se mais evidentes, quando se constata que $72 \%$ das laqueaduras realizadas no estado de São Paulo o foram por ocasião de cesáreas, momento inadequado para esta opção ${ }^{4}$. A laqueadura é realizada muitas vezes sem maior critério e sem a devida consideração de sua irreversibilidade ${ }^{21}$. Além de todos estes fatos, devese considerar ainda que os custos médicos, sociais e econômicos de uma cesárea indicada exclusivamente para realizar-se uma laqueadura implicam a anulação de possiveis investimentos do sistema de saúde em outras áreas carentes de re$\operatorname{cursos}^{1,6}$.

Os dados relatados pela PNDS de 1996, em São Paulo, mostrara baixa freqüência de métodos anticoncepcionais transitórios, totalizando 10\% de freqüência de uso, sendo que o uso do condom representou 7\% das mulheres entre 15 e 49 anos $^{3}$. No presente estudo, a utilização de condom foi relatada em $28,1 \%$. O fato de o condom ser o segundo método transitório mais utilizado reflete não apenas maior participação masculina na anticoncepção, mas pode estar refletindo maior preocupação da sociedade com a questão da $\operatorname{AIDS}^{13-15}$.

Um dos principais problemas relacionado à elevada porcentagem de usuárias de pílula são os fatores de risco para seu uso. Esta situação se torna grave quando não ocorre indicação médica para este uso, situação esta relatada em alguns estudos, nos quais se observa que nem sempre há a 
indicação por profissional da área de saúde ${ }^{3,16}$. No presente estudo, este percentual foi relativamente elevado, chegando a $74 \%$ das entrevistadas. Portanto, nem sempre a falha é da usuária, que não se submete à consulta médica, mas do serviço de saúde que se omite em discutir o problema da anticoncepção quando a mulher realiza a referida consulta, ressaltando que faz parte da responsabilidade do profissional que orienta a usuária atuar na detecção preventiva dos fatores de risco para uso dos contraceptivos. Para utilização do método natural, as entrevistadas receberam orientações dos profissionais de saúde em $42,3 \%$ das vezes, para utilização do condom, receberam, na mesma proporção, informações de parentes ou amigos e profissionais da área de saúde em 31,2\% das vezes. Paralelamente à presença de fatores de risco, relacionados ao uso da pílula e demais métodos, configuram-se efeitos secundários que podem levar à interrupção do método e gerar seu uso inadequado ${ }^{12,20,21}$.

Em relação à interrupção do uso dos métodos contraceptivos, os motivos pesquisados para a interrupção foram baseados nas cinco categorias de Curtis e Hammerslough ${ }^{20}$ e adaptados para este estudo. Os principais motivos de interrupção encontrados foram o desejo de gravidez, principalmente naquelas mulheres que se utilizavam de métodos naturais, devido à realização de laqueadura naquelas que se utilizavam do condom, e devido a efeitos colaterais do método naquelas que se utilizavam de pílula. Vale pormenorizar que dentre as mulheres entrevistadas que interromperam o uso da pílula anticoncepcional, os motivos foram devido a efeitos colaterais do uso da pílula em $23,8 \%$ e realização da laqueadura em $16,8 \%$ das entrevistadas que utilizavam o método. Estes achados são semelhantes aos encontrados em estudo realizado na região Nordeste do Brasil, em 1991, onde se observou que a interrupção do uso de pílula foi decorrente de efeitos colaterais em $19,7 \%{ }^{20}$.

Em relação ao uso da pílula anticoncepcional, grande parte dos estudos mostram alta prevalência ${ }^{3,12,20}$. O fato de a pílula anticoncepcional ser o método transitório mais utilizado pela população estudada não tem significado necessariamente positivo ou negativo. O mais importante, qualquer que seja o método anticoncepcional, é avaliar se as mulheres que o utilizam podem realizálo ou não ${ }^{22}$.

Desta forma, é importante a identificação das contra-indicações absolutas e relativas para o uso de pílula. Existem fatores de risco entre usuárias desse método que podem resultar em complicações graves e até óbito, principalmente na ausência de acompanhamento médico ${ }^{3,23}$. Em se tratando de contra-indicações ou fatores de risco para o uso de pílula anticoncepcional, observou-se que cerca de metade das mulheres entrevistadas não apresentaram nenhum fator de risco, ao passo que outras apresentavam mais de um fator de risco, sendo o mais freqüente o tabagismo, constatado em $23,3 \%$ das vezes. É importante considerar-se a limitação do presente estudo quanto a caracterizar com rigor as contra-indicações absolutas e relativas ao uso dos contraceptivos, principalmente a pílula anticoncepcional. Dentre estas, sabe-se que o tabagismo, que foi a mais prevalente, pode não ser considerado contra-indicação em mulheres jovens. Guando analisadas as variáveis interdependentes como a idade e hábito de fumar entre as usuárias de pílula, como aplicado neste estudo, observou-se que a maioria, $56,4 \%$ destas mulheres, estava na faixa etária acima de 35 anos, justamente a faixa que passa a ser considerada de risco $^{24,25}$.

O problema do uso inadequado e da ausência de orientação médica para o uso da pílula agrava-se quando se verifica que um total de 29,6\% das usuárias do método o estão utilizando por mais de cinco anos e apenas aproximadamente a terça parte das mulheres $(35,9 \%)$ se submeteram à consulta prévia para o início do uso.

A análise deste estudo mostra como conclusões gerais: elevadas taxas de laqueadura (32\%), expressivo uso de métodos transitórios tipo condom $(28,1 \%)$, não-uso de métodos ditos modernos, como endoceptivos e implantes, e detecção de usuárias de pílulas com contra-indicações, embora não absolutas, com mais de 5 anos de uso.

Desde a divulgação no final da década de 1980 do marco conceitual, amplamente utilizado como referência para qualidade de atenção em planejamento familiar, contendo 6 elementos fundamentais (escolha livre de métodos, informação para as usuárias, competência técnica, relação usuária-serviço, acompanhamento de usuárias e integração do planejamento familiar ao atendimento em saúde reprodutiva) ${ }^{12}$, muito se tem reinvidicado para alcançar o planejamento familiar dentro desta proposta. No entanto, a análise tanto de programas de menor abrangência, como também em âmbito nacional, revela inadequado uso de métodos anticoncepcionais como conseqüência da desinformação da população associada à dificuldade de acesso aos serviços de saúde de qualidade e aos próprios métodos ${ }^{12-16}$. Portanto, caso não se promova a conscientização da classe médica, melhoria de acesso à informação às mulheres e melhor posicionamento nos programas e ações do Ministério da Saúde e entidades envolvi- 
das, haverá progressiva piora nos indicadores em planejamento familiar refletidos na qualidade de vida e saúde reprodutiva da mulher.

\section{Referências}

1. Martins-Costa S, Ramos JGL. A questão das cesarianas. Rev Bras Ginecol Obstet. 2005;27(10):571-4

2. Population growth and family planning: six billion and beyond. Contracept Rep. 2000;10(6):8-14.

3. Vieira EM, Badiani R, Fabbro ALD, Rodrigues Junior AL. Características do uso de métodos anticoncepcionais no Estado de São Paulo. Rev Saúde Pública. 2002;36(3):263-70

4. Faúndes A, Costa RG, Pádua KS, Perdigão AM. Associação entre prevalência de laqueadura tubária e características sócio-demográficas de mulheres e seus companheiros no Estado de São Paulo, Brasil. Cad Saúde Pública. 1998;14 Supl 1:49-57.

5. Fagundes ML, Mendes MC, Patta MC, Moura MD, Campos $\mathrm{AD}$, Duarte $\mathrm{G}$, et al. Padrão endometrial em mulheres submetidas à laqueadura tubária. Rev Bras Ginecol Obstet. 2005;27(9):529-33.

6. Moraes MS, Goldenberg P. Cesáreas: um perfil epidêmico. Cad Saúde Pública. 2001;17(3):509-19.

7. Theme-Filha MM, Silva RI, Noronha CP. Mortalidade materna no município do Rio de Janeiro, 1993 a 1996. Cad Saúde Pública. 1999;15(2):397-403.

8. Laurenti R. Marcos referenciais para estudos e investigações em mortalidade materna. Cad Saúde Pública. 1988;22(6):507-12.

9. Costa AAR, Ribas MSSS, Amorim MMR, Santos LC. Mortalidade Materna na cidade do Recife. Rev Bras Ginecol Obstet. 2002;24(7):455-62

10. Ministério da Saúde. Secretaria de Atenção à Saúde. Departamento de Ações Programáticas Estratégicas. Política nacional de atenção integral à saúde da mulher: princípios e diretrizes. Brasília; 2004. p. 33.

11.Aquino EML, Heilborn ML, Knauth D, Bozon M, Almeida MC, Araújo J, et al. Adolescência e reprodução no Brasil: a heterogeneidade dos perfis sociais. Cad Saúde Pública. 2003;19 Supl 2:377-88.

12. Osis MJD, Duarte, GA, Crespo ER, Espejo X, Pádua KS. Escolha de métodos contraceptivos entre usuárias de um serviço público de saúde. Cad Saúde Pública. 2004;20(6):1586-94.

13. Martins LBM, Costa-Paiva L, Osis MJD, Sousa MH, Pinto Neto AM, Tadini V. Conhecimento sobre métodos anticoncepcionais por estudantes adolescentes. Rev Saúde Pública. 2006;40(1):57-64.

14. Magalhães J, Rossi AS, Amaral E. Uso de condom feminino por mulheres infectadas pelo HIV. Rev Bras Ginecol Obstet. 2003;25(6):389-95.

15. Paniz VMV, Fassa AG, Silva MC. Conhecimento sobre anticoncepcionais em uma população de 15 anos ou mais de uma cidade do Sul do Brasil. Cad Saúde Pública. 2005;21(6):1747-60.

16. Moura ERF, Silva RM. Competência profissional e assistência em anticoncepção. Rev Saúde Pública. 2005;39(5):795-801.

17. Instituto Brasileiro de Geografia e Estatística-IBGE. Estimativas de população para o Brasil em 2003. Brasília: Ministério da Saúde/Datasus; 2003.

18.Jekel JF, Katz D, Elmore J. Epidemiologia, estatística e medicina preventiva. Porto Alegre: Artmed; 2005.

19. Associação Brasileira de Empresas de PesquisasABEP [homepage da Internet]. Critério de classificação econômica Brasil (CCEB). 2004 [citado 2005 Dez 17]. Disponivel em: http://www.abep.org

20.Leite IC. Descontinuação de métodos anticoncepcionais no Nordeste do Brasil, 1986 1991. Cad Saúde Pública. 2003;19(4):1005-16.

21. Carvalho MLO, Schor N. Motivos de rejeição aos métodos contraceptivos reversiveis em mulheres esterelizadas. Rev Saúde Pública. 2005;39(5):78894.

22. World Health Organization-WHO [homepage da Internet]. Medical eligibility criteria for contraceptive use. 2nd ed. Geneve: WHO; 2004 [cited 2006 Jan 22]. Available from: http://www.who.int/ reproductive $=$ health

23. Curtis KM, Chrisman CE, Peterson HB; WHO Programme for Mapping Best Practices in Reproductive Health. Contraception for women in selected circumstances. Obstet Gynecol. 2002;96(6):1100-12.

24. World Health Organization-WHO. Cardiovascular disease and steroid hormone contraception. Geneva; 1998. [WHO Technical Report Series, 877].

25. Neinstein L. Contraception in women with special medical needs. Compr Ther. 1998;24(5):229-50. 